\title{
NOUVELle
}

\section{Rôle des récepteurs NMDA et du glutamate astrocytaire dans la neurogenèse postnatale}

Jean-Claude Platel, Angélique Bordey
J.C. Platel : University of Saarland, School of Medicine, Department of Physiology, Kirrberger Strasse, Buildings 58, 66424 Homburg/Saar, Allemagne.

jc.platel@gmail.com

A. Bordey: Departments of Neurosurgery, and Cellular and Molecular Physiology, Yale University School of Medicine, 333 Cedar Street, FMB 422, New Haven,

CT 06520-8082, États-Unis.

angelique.bordey@yale.edu
> Jusqu'à très récemment, on pensait que le cerveau ne fabriquait des neurones que pendant la période embryonnaire. Il est maintenant clairement démontré que certaines zones restreintes du cerveau contiennent des cellules souches neuronales et conservent la remarquable possibilité de donner naissance à de nouveaux neurones tout au long de la vie adulte [1]. La neurogenèse persiste essentiellement dans deux régions: la zone sous-granulaire (ZSG) du gyrus denté de l'hippocampe et la zone sousventriculaire (ZSV) qui borde les ventricules latéraux. Pour donner une idée de l'ampleur de la neurogenèse, on estime chez le rat de 10000 à 30000 le nombre de nouveaux neurones produits chaque jour dans la ZSV. Ces neurones migrent rostralement (rostral signifie vers le nez, du latin rostrum : bec) sur plusieurs millimètres par une voie qu'on appelle la voie de migration rostrale (VMR) pour s'intégrer dans le réseau préexistant du bulbe olfactif. La fonction de ces neurones reste inconnue chez l'homme, mais la présence de cellules souches représente un énorme potentiel clinique. Il est donc crucial de découvrir les mécanismes régulant la neurogenèse chez les mammifères, ce qui ouvrirait la voie à son amplification pour réparer le cerveau ou à sa diminution si ces cellules souches deviennent cancéreuses.

Les mécanismes de régulation associent un programme génétique et des signaux en provenance du microenvironnement cellulaire, et nous nous sommes intéressés à ces signaux et plus particulièrement à l'un d'entre eux, le neurotransmetteur glutamate. Le glutamate est le principal neurotransmetteur excitateur du système nerveux adulte. Son rôle est important lors du développement cérébral embryonnaire, et I'un de ses récepteurs, le récepteur $\mathrm{N}$-méthyl-D-aspartate (NMDA) joue un rôle déterminant dans la survie des nouveaux neurones dans l'hippocampe [2]. Au niveau de la ZSV, la signalisation glutamatergique semblait n'apparaître que très tardivement, une fois les cellules sur le point de s'intégrer dans le bulbe olfactif. Néanmoins nos études précédentes démontraient la présence et la fonctionnalité d'autres récepteurs au glutamate durant la migration rostrale [3]. Nous avons donc entrepris de caractériser plus en détails l'apparition des récepteurs NMDA, la source du glutamate qui les active ainsi que le rôle de ces récepteurs en utilisant la technique knock-out sur cellule unique in vivo [4].

\section{Expression des récepteurs NMDA sur} les neuroblastes émergeant de la ZSV Nous avons tout d'abord analysé l'expression des récepteurs NMDA lors de la phase de migration/maturation des neuroblastes dans la ZSV et la VMR. Nous avons pour cela utilisé deux techniques, l'électrophysiologie et l'imagerie calcique, que nous avons appliquées à des tranches de cerveau. L'électrophysiologie permet d'enregistrer les courants qui passent au travers de la membrane cellulaire et elle possède la sensibilité suffisante pour identifier la présence d'un seul canal. L'imagerie calcique utilise une sonde calcique fluorescente qui est introduite dans une population de cellules et sert à mesurer des changements de la concentration intracellulaire des ions calcium comme par exemple lors de l'ouverture des récepteurs NMDA. Ces deux techniques nous ont permis d'identifier l'expression de récepteurs NMDA très tôt lors de la production des neuroblastes (précurseurs des neurones nouvellement produits), peu de temps après leur naissance dans la ZSV. La densité de ces canaux augmente progressivement lors de leur maturation vers le bulbe olfactif. L'électrophysiologie nous a également permis d'identifier une activité électrique spontanée due à l'activation de ces récepteurs NMDA. Ce résultat est très important puisqu'il signifie que ces récepteurs sont fonctionnels et qu'ils sont activés par le glutamate présent dans la ZSV. Nous avons donc cherché quelle était la source du glutamate dans la ZSV.

\section{Le glutamate sécrété}

\section{par les astrocytes de la ZSV}

protège les neuroblastes de l'apoptose

La plupart des neuroblastes contiennent et libèrent le neurotransmetteur GABA (acide $\gamma$-aminobutyrique); il est donc peu probable qu'ils libérent également du glutamate. Outre les neuroblastes, une deuxième population de 
cellules dans la ZSV est représentée par des cellules de type astrocytes qu entourent les neuroblastes (Figure I), certains d'entre eux étant les cellules souches [8]. Les astrocytes du cerveau (hors ZSV) libèrent du glutamate mais la fonction et le mode de libération de ce glutamate restent controversés $[5,6]$. Nous avons donc testé I'hypothèse selon laquelle les astrocytes de la ZSV pouvaient produire et libérer du glutamate qui, ensuite, activerait les récepteurs NMDA présents sur les neuroblastes. Nous avons tout d'abord réalisé une série de marquages immunohistologiques et en microscopie électronique avec différents marqueurs caractéristiques des cellules libérant le glutamate. Tous ces marqueurs: le glutamate lui-même, les transporteurs vésiculaires du glutamate de type 1 , la synaptobrévine 2 (un marqueur de libération vésiculaire), qui sont classiquement exprimés par les neurones glutamatergiques, sont présents également dans les astrocytes de la ZSV. Dans un second temps, pour déterminer si cette machinerie de production était fonctionnelle, nous avons utilisé une souris transgénique conçue par K.D. McCarthy dans laquelle un récepteur couplé aux protéines $G$ appelé MrgAl (mas-related gene) est exprimé spécifiquement dans les astrocytes. Ce récepteur peut être spécifiquement activé par l'application d'un peptide, ce qui déclenche une augmentation de calcium spécifiquement dans les astrocytes. Nous avons stimulé les astrocytes de la ZSV avec ce peptide tout en enregistrant

A

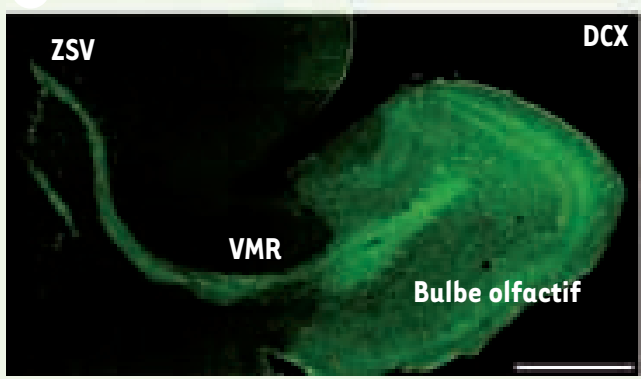

par électrophysiologie l'activité des récepteurs NMDA dans les neuroblastes. Nous avons ainsi constaté que le peptide entraînait une augmentation de calcium dans les astrocytes de la ZSV suivie d'une libération de glutamate suffisante pour activer les récepteurs NMDA dans les neuroblastes. Nous avons finalement cherché à comprendre quel était le rôle des récepteurs NMDA dans la neurogenèse plus particulièrement sur la survie et la migration des neuroblastes. Nous avons tout d'abord injecté in vivo un antagoniste des récepteurs NMDA, le MK801. Dès deux heures après l'injection de MK80l on constatait un nombre de neuroblastes apoptotiques plus important que chez les animaux contrôles.

\section{Identification}

du rôle des récepteurs NMDA

in vivo dans les cellules de la ZSV

Pour confirmer que le blocage des récepteurs NMDA induisait la mort des neuroblastes, nous avons éliminé les récepteurs NMDA via une stratégie d'électroporation postnatale in vivo [7]. Cette technique consiste à injecter un plasmide contenant un gène d'intérêt dans l'un des ventricules latéraux, et, par l'administration d'impulsions électriques, de le faire entrer dans les cellules de la ZSV bordant le ventricule. Nous avons utilisé cette technique chez des animaux dont le gène codant pour l'une des sous-unités du récepteur NMDA (la sous unité NRl) est flanqué par des séquences loxP. Un plasmide exprimant la recombinase Cre a été électroporé in vivo, ce qui entraîne l'excision du gène entre les 2 sites loxp et de la séquence stop bloquant l'expression d'un second gène codant pour une étiquette fluorescente (Figure 2). Cette technique - également appelé knock-out sur cellule unique - permet d'enlever un gène précis dans une cellule précise sans affecter le reste du cerveau ni même les cellules non électroporées adjacentes. Grâce à cette approche, nous avons pu confirmer que l'absence de récepteurs NMDA ne changeait pas la vitesse de migration des neuroblastes alors qu'elle entraînait la mort de plus de $30 \%$ des cellules dans la VMR, c'est-à-dire bien avant que ces cellules commencent à s'intégrer dans le bulbe olfactif.

\section{Conclusion}

Ce travail a permis de révéler pour la première fois le rôle critique des récepteurs NMDA dans la neurogenèse survenant après la naissance. II a également mis en évidence un nouveau type d'interaction entre les astrocytes de la ZSV et les neuroblastes, les astrocytes de la ZSV pouvant, via leur libération de glutamate, permettre aux nouveaux neurones exprimant des récepteurs NMDA de survivre. Enfin, il faut souligner que les gènes (GRIN1, GRIN2) codant pour le récepteur NMDA sont anormaux (mutés) dans certaines maladies neurodéveloppementales et certains troubles psychiatriques comme la schizophrénie [9] (pour une réference voir: http://geneticassociationdb.nih.gov) ou le trouble déficit de l'attention/hyperactivité (TDA/H) [10]

Figure 1. Structure de la zone sous-ventriculaire. A. Reconstruction d'une tranche sagittale d'un cerveau de 25 jours marquée dont les neuroblastes expriment la doublecortine. Les chaînes de neuroblastes de la ZSV convergent pour former la voie de migration rostrale qui se termine dans le bulbe olfactif. Barre d'échelle: $500 \mu \mathrm{m}$. B. Schéma simplifié illustrant l'organisation cellulaire de la ZSV. Les prolongements des astrocytes de la ZSV entourent un groupe de neuroblastes. DCX: double cortine. 


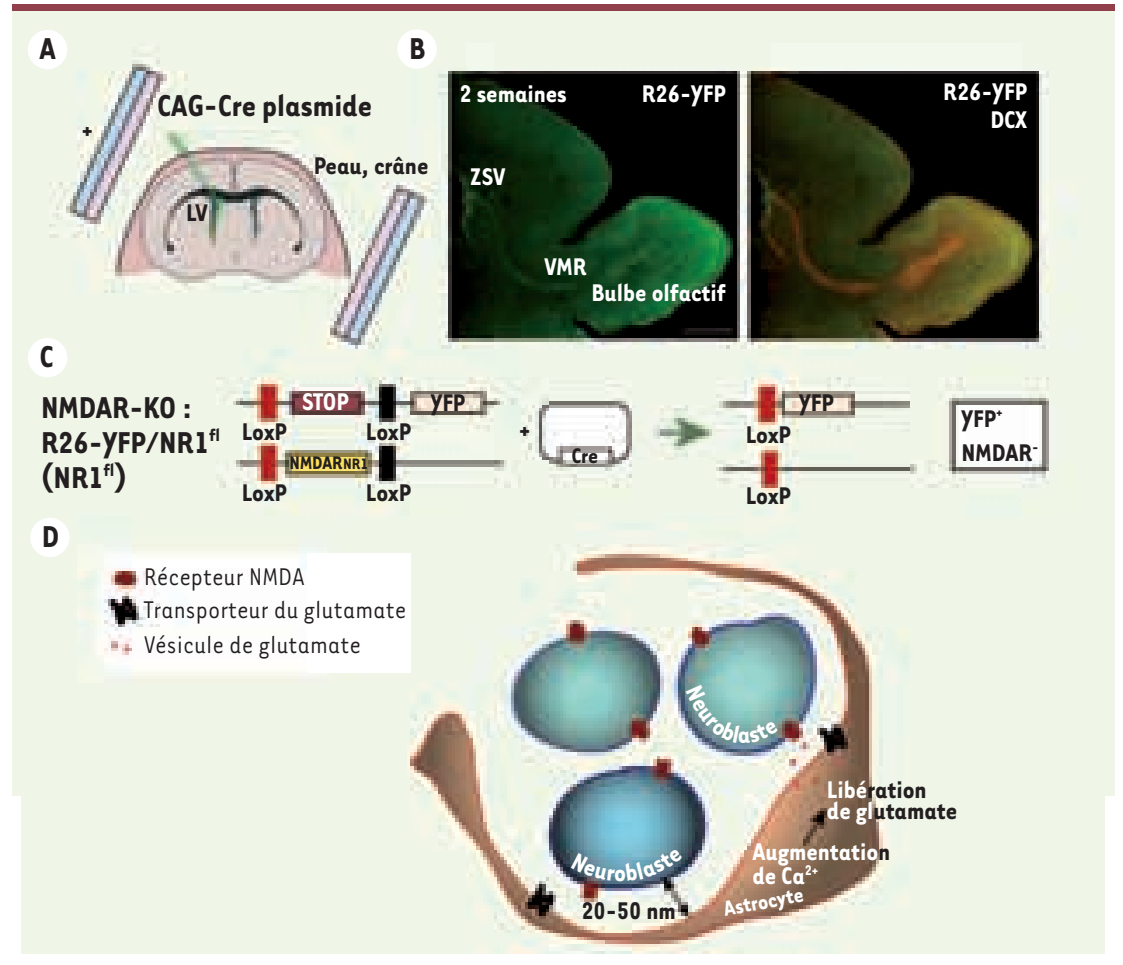

Figure 2. Méthode du knock-out sur cellule unique mettant en évidence le rôle de la signalisation glutamatergique dans la ZSV. A. Diagramme illustrant la méthode d'électroporation in vivo. B. Exemple d'une image confocale d'une tranche sagittale à 14 jours après électroporation (marquage $\mathrm{yFP}^{+}$, yellow fluorescent protein) et marquée pour la doublecortine (DCX). C. Diagramme illustrant l'effet de l'expression de la recombinase Cre dans les cellules électroporées dans des animaux Rosa-26-YFP et NR1-flox (récepteur du glutamate). D. Schéma simplifié illustrant la signalisation glutamatergique dans la ZSV. Après une augmentation de calcium, les astrocytes de la ZSV peuvent libérer du glutamate qui va activer les récepteurs NMDA exprimés sur les neuroblastes, leur permettant ainsi de survivre.

ce qui pourrait contribuer a certains des symptômes de ces patients. Comprendre comment la perte des récepteurs NMDA affecte la construction d'un réseau neuronal pourrait permettre de mieux appréhender les mécanismes de ces troubles neurologiques. $\diamond$

Function of NMDA receptors activated by astrocytic glutamate on postnatal neurogenesis

\section{CONFLIT D'INTÉRÊTS}

Les auteurs déclarent n'avoir aucun conflit d'intérêts concernant les données publiées dans cet article.

\section{RÉFÉRENCES}

1. De Chevigny A. Lledo PM. La neurogenèse bulbaire et son impact neurologique. Med Sci (Paris) 2006; $22: 607-13$.

2. Tashiro A, Sandler VM, Toni N, et al. NMDA-receptormediated, cell-specific integration of new neurons in adult dentate gyrus. Nature 2006 ; 442 : 929-33.

3. Platel JC, Heintz T, Young $S$, et al. Tonic activation of GLUK5 kainate receptors decreases neuroblast migration in whole-mounts of the subventricular zone. J Physiol 2008 ; 586 : 3783-93.

4. Platel JC, Dave KA, Gordon V, et al. NMDA receptors activated by subventricular zone astrocytic glutamate are critical for neuroblast survival prior to entering a synaptic network. Neuron $2010 ; 65: 859-72$.

5. Bezzi P, Gundersen V, Galbete JL, et al. Astrocytes contain a vesicular compartment that is competent for regulated exocytosis of glutamate. Nat Neurosci $2004 ; 7: 613-20$
6. Agulhon C, Fiacco TA, McCarthy KD. Hippocampal short-and long-term plasticity are not modulated by astrocyte $\mathrm{Ca}^{2+}$ signaling. Science $2010 ; 327$ : 1250-4.

7. Boutin C, Diestel S, Desoeuvre A, et al. Efficient in vivo electroporation of the postnatal rodent forebrain. PLoS One 2008 ; 3 : el 883.

8. Spassky N, Caillé I. La niche neurogénique adulte entre dans la troisième dimension. Med Sci (Paris) $2009 ; 25: 17-8$

9. Krebs MO. Intégrité chromosomique et schizophrénie. Med Sci (Paris) 2009; $25: 219-21$.

10. Purper-Ouakil D, Lepagnol-Bestel AM, Grosbellet $\varepsilon$, et al. Neurobiologie du trouble déficit de l'attention/ hyperactivité. Med Sci (Paris) $2010 ; 26: 487-96$.

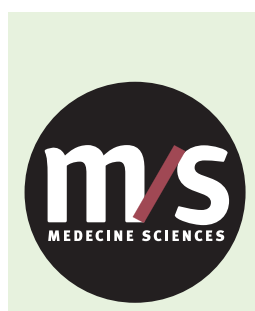

$>$ Grâce à $m / s$, vivez en direct les progrès Tarifs d'abonnement M/S - 2010 des sciences biologiques et médicales

Abonnez-vous

à Médecine/Sciences
Bulletin d'abonnement page 674 dans ce numéro de $\mathrm{m} / \mathrm{s}$

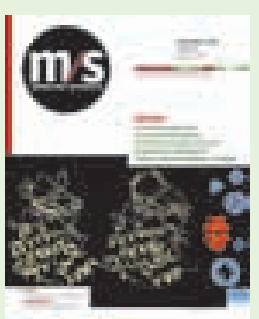

